\title{
Beyond Beck's Triad: A Rare Cause of Cardiac Tamponade and Hemoptysis
}

\author{
Homam Moussa Pacha, MD, ${ }^{1}$ Mohamad Soud, MD, ${ }^{1}$ M. Chadi Alraies, MD ${ }^{2}$ \\ ${ }^{1}$ Department of Internal Medicine, MedStar Washington Hospital Center, Washington, DC ${ }^{2}$ Wayne State University, Detroit Medical Center \\ Heart Hospital, Detroit, MI
}

\begin{abstract}
Background: Primary cardiac angiosarcoma (CAS) is a rare tumor with a dismal prognosis. Many patients present with noncardiac symptoms related to metastatic disease that could delay the diagnosis and deteriorate the outcome.

Case Report: A 36-year-old male presented with hemoptysis. Initial imaging and biopsies were inconclusive, but a repeat transthoracic echocardiogram to evaluate the patient's pericardial effusion showed a mass inferior to the right atrium and invading the wall. Biopsy results from 3 different sites confirmed the diagnosis of CAS. However, the patient's course was complicated with respiratory failure that ultimately led to his death. Autopsy demonstrated CAS involving the full thickness of the right atrium and pericardial tissue, with a diffuse metastatic process involving the lung, brain, and stomach.

Conclusion: The case highlights the importance of having an increased level of suspicion for cardiac malignancy among young patients presenting with noncardiac symptoms.
\end{abstract}

Keywords: Heart neoplasms, hemoptysis, sarcoma

Address correspondence to M. Chadi Alraies, Wayne State University, Detroit Medical Center Heart Hospital, 311 Mack Ave., Detroit, MI 48201 Tel: (216) 255-0008. Email: alraies@hotmail.com

\section{INTRODUCTION}

Primary cardiac tumors are rare, found in $0.02 \%$ of autopsies. ${ }^{1}$ While the majority of primary cardiac tumors are benign, $25 \%$ are malignant. ${ }^{2}$ Sarcomas account for $95 \%$ of malignant cardiac tumors, and one-third of them are cardiac angiosarcomas (CASs), the most common subtype. ${ }^{3,4}$ CASs are located predominantly in the right atrium, ${ }^{2}$ and patients commonly present with pericardial effusion with or without tamponade. $^{5}$ In 1935, Dr Claude Beck described 2 triads of symptoms that aid in the diagnosis of cardiac tamponade: the acute tamponade triad (hypotension, venous distension, and diminished heart sounds) and the chronic compression triad (high venous pressure, ascites, and diminished heart sounds). ${ }^{6}$ Nevertheless, not uncommonly, CAS initially presents with noncardiac clinical symptoms, and in many cases, CAS is discovered on autopsy. ${ }^{2}$

\section{CASE REPORT}

A 36-year-old male presented with progressive dyspnea and hemoptysis for 2 weeks. Physical examination was remarkable for low-grade fever, distant heart sounds, and elevated jugular venous pressure. Chest $\mathrm{x}$-ray showed multiple pulmonary nodules with right pleural effusion (Figure, A). Computed tomography (CT) of the chest revealed bilateral innumerable diffuse pulmonary nodules and large pericardial effusion (Figure, B). Transthoracic echocardiogram (TTE) was remarkable for large pericardial effusion with right ven- tricular and atrial diastolic compression, indicating early tamponade (Figure, C). Urgent pericardiocentesis removed $1,500 \mathrm{cc}$ of bloody pericardial fluid. Fluids analysis showed an exudative fluid with inflammatory cells with cytology negative for malignancy or infection. Extensive rheumatologic and infectious workups were negative. CT-guided and endobronchial ultrasound-guided biopsy were inconclusive. Repeat TTE to evaluate the pericardial effusion showed a mass inferior to the right atrium and invading the wall that was not seen on the previous TTE (Figure, D). Given this new echocardiographic finding and the patient's clinical presentation, suspicion of metastatic lung nodules secondary to primary cardiac tumor was high. The patient was referred for video-assisted thoracoscopic surgery. Biopsy results from 3 different sites confirmed the diagnosis of CAS. However, the patient's course was complicated with respiratory failure that ultimately led to his death. Autopsy demonstrated CAS involving the full thickness of the right atrium and pericardial tissue, with a diffuse metastatic process involving the lung, brain, and stomach.

\section{DISCUSSION}

CAS is a fatal tumor that has nonspecific presentations other than the typical presentations of pericardial effusion and right-sided heart failure; hemoptysis is one of the nonspecific symptoms. ${ }^{5,7}$ Many patients present with noncardiac symptoms related to metastatic disease involving the 

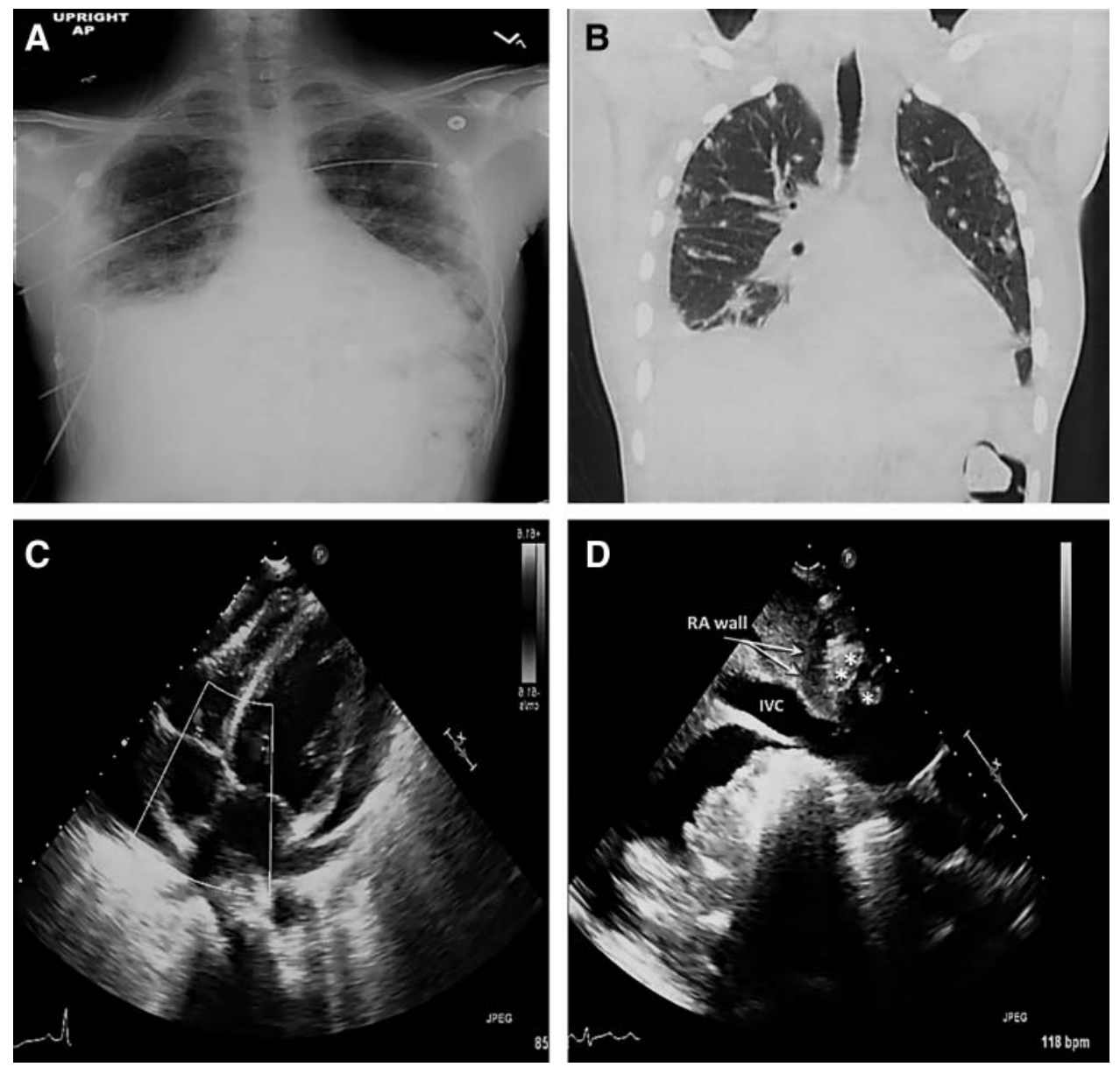

Figure. A: Chest x-ray shows multiple pulmonary nodules with right pleural effusion. B: Computed tomography of the chest shows bilateral innumerable diffuse pulmonary nodules and large pericardial effusion. C: Transthoracic echocardiogram (TTE) shows large pericardial effusion with signs suggesting early tamponade. D: Repeat TTE shows a mass invading the right atrium (RA) wall (arrows and asterisks). IVC, inferior vena cava.

lung, liver, bones, and other organs. ${ }^{4,5,8}$ A series of $18 \mathrm{CAS}$ cases showed pericardial effusion to be the most common presentation. ${ }^{5}$ Median overall survival was 13 months in the entire cohort; among patients with CAS, median survival was 6 months compared to 19.5 months for patients with a localized tumor. ${ }^{5}$ Because of the tendency for early metastasis, CAS has a poor prognosis, with median survival dependent on the extent of the disease and response to therapy. $4,5,8$

Echocardiogram sensitivity in detecting CAS can reach $75 \%$; $^{9}$ advanced imaging such as cardiac magnetic resonance imaging adds great value in differentiating between benign and malignant masses. ${ }^{10}$ However, a tissue biopsy is needed to confirm the diagnosis, either open biopsy for operable tumors or transvenous endomyocardial biopsy for inoperable tumors.

Multimodality therapies for CAS-including surgical resection, radiotherapy, and chemotherapy-are evolving, and the lack of a defined systemic therapy is attributed to the rarity of the disease. ${ }^{4,5}$ Complete surgical resection of localized disease is considered the most effective treatment and has been shown to improve survival. ${ }^{4,5,8} \mathrm{~A}$ study of 13 patients with CAS demonstrated that combined therapy of surgical resection and chemoradiotherapy had the best prognosis. ${ }^{8}$ The use of radiotherapy in CAS is challenging because of the risk of radiotherapy-induced cardiac toxicity in the form of pericarditis and cardiomyopathy. ${ }^{11}$

\section{CONCLUSION}

Primary CAS is a rare and challenging tumor with a dismal prognosis. Metastasis with nonspecific symptoms as the initial presentation is not uncommon. Many patients present with symptoms related to metastatic disease that could delay the diagnosis and deteriorate the outcome despite multimodality imaging.

\section{ACKNOWLEDGMENTS}

The authors have no financial or proprietary interest in the subject matter of this article.

\section{REFERENCES}

1. Reynen K. Frequency of primary tumors of the heart. Am J Cardiol. 1996 Jan 1;77(1):107. 
2. Silverman NA. Primary cardiac tumors. Ann Surg. 1980 Feb;191 (2):127-138.

3. Kodali $D$, Seetharaman K. Primary cardiac angiosarcoma. Sarcoma. 2006;2006:39130.

4. Isambert N, Ray-Coquard I, Italiano A, et al. Primary cardiac sarcomas: a retrospective study of the French Sarcoma Group. Eur J Cancer. 2014 Jan;50(1):128-136. doi: 10.1016/j. ejca.2013.09.012.

5. Look Hong NJ, Pandalai PK, Hornick JL, et al. Cardiac angiosarcoma management and outcomes: 20-year singleinstitution experience. Ann Surg Oncol. 2012 Aug;19(8):27072715. doi: 10.1245/s10434-012-2334-2.

6. Beck CS. Two cardiac compression triads. JAMA. 1935;104 (9):714-716. doi: 10.1001/jama.1935.02760090018005.

7. Agaimy A, Rösch J, Weyand M, Strecker T. Primary and metastatic cardiac sarcomas: a 12-year experience at a German heart center. Int J Clin Exp Pathol. 2012;5(9):928-938.
8. Fatima J, Duncan AA, Maleszewski JJ, et al. Primary angiosarcoma of the aorta, great vessels, and the heart. J Vasc Surg. 2013 Mar;57(3):756-764. doi: 10.1016/j.jvs.2012.09.023.

9. Kupsky DF, Newman DB, Kumar G, Maleszewski JJ, Edwards WD, Klarich KW. Echocardiographic features of cardiac angiosarcomas: the Mayo Clinic experience (1976-2013). Echocardiography. 2016 Feb;33(2):186-192. doi: 10.1111/ echo. 13060.

10. Esposito A, De Cobelli F, Ironi G, et al. CMR in the assessment of cardiac masses: primary malignant tumors. JACC CardiovasC Imaging. 2014 Oct;7(10):1057-1061. doi: 10.1016/j. jcmg.2014.08.002.

11. Prosnitz RG, Chen YH, Marks LB. Cardiac toxicity following thoracic radiation. Semin Oncol. 2005 Apr;32(2 Suppl 3):S71S80.

This article meets the Accreditation Council for Graduate Medical Education and the American Board of Medical Specialties Maintenance of Certification competencies for Patient Care and Medical Knowledge. 